\title{
Role of nitric oxide and superoxide in Giardia lamblia killing
}

P.D. Fernandes and J. Assreuy
Departamento de Farmacologia Básica e Clínica, Instituto de Ciências Biomédicas, Centro de Ciências da Saúde, Universidade Federal do Rio de Janeiro, 21944-970 Rio de Janeiro, RJ, Brasil

\author{
Correspondence \\ J. Assreuy \\ Departamento de Farmacologia \\ ICBS/CCS \\ Universidade Federal do \\ Rio de Janeiro \\ Caixa Postal 68013 \\ 21944-970 Rio de Janeiro, RJ \\ Brasil \\ Fax: 55 (021) 270-8647 \\ E-mail: assreuy@server.bioqmed.ufr.br \\ P.D. Fernandes and J. Assreuy are \\ recipients of CNPq fellowships. \\ Research supported by Fundação \\ Universitária José Bonifácio/UFRJ/ \\ Brasil.

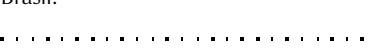

Received April 23, 1996

Accepted October 2, 1996

\begin{abstract}
Giardia lamblia trophozoites were incubated for $2 \mathrm{~h}$ with activated murine macrophages, nitric oxide (NO) donors or a superoxide anion generator $(20 \mathrm{mU} / \mathrm{ml}$ xanthine oxidase plus $1 \mathrm{mM}$ xanthine). Activated macrophages were cytotoxic to Giardia trophozoites ( $60 \%$ dead trophozoites). This effect was inhibited (>90\%) by an NO synthase inhibitor $(200 \mu \mathrm{M})$ and unaffected by superoxide dismutase (SOD, $300 \mathrm{U} / \mathrm{ml}$ ). Giardia trophozoites were killed by the NO donors, S-nitroso-acetyl-penicillamine (SNAP) and sodium nitroprusside (SNP) in a dose-dependent manner $\left(\mathrm{LD}_{50} 300\right.$ and $50 \mu \mathrm{M}$, respectively). A dual NO-superoxide anion donor, 3-morpholino-sydnonimine hydrochloride (SIN-1), did not have a killing effect in concentrations up to $1 \mathrm{mM}$. However, when SOD $(300 \mathrm{U} / \mathrm{ml})$ was added simultaneously with SIN-1 to Giardia, a significant trophozoite-killing effect was observed ( $\sim 35 \%$ dead trophozoites at $1 \mathrm{mM})$. The mixture of SNAP or SNP with superoxide anion, which yields peroxynitrite, abolished the trophozoite killing induced by NO donors. Authentic peroxynitrite only killed trophozoites at very high concentrations $(3 \mathrm{mM})$. These results indicate that NO accounts for Giardia trophozoite killing and this effect is not mediated by peroxynitrite.
\end{abstract}

Key words

- Nitric oxide

- Macrophage

- Peroxynitrite

- Giardia lamblia

- Cytotoxicity

- Superoxide

\section{Introduction}

When macrophages are activated, they become competent to kill several pathogens and to lyse tumor cells. During the activation process, macrophages produce an array of biologically active molecules that includes tumor necrosis factor-alpha (TNF- $\alpha$ ), interleukin-1 (IL-1) and reactive oxygen intermediates such as superoxide and hydrogen peroxide (1).

Recent studies have shown that activated macrophages also produce large amounts of an unstable gas, nitric oxide (NO; Ref. 2). Nitric oxide is synthesized from L-arginine by the enzyme NO synthase (NOS), which occurs in three forms. The endothelial NOS (eNOS) is known to play a role in physiological vasodilatation whereas neuronal NOS (nNOS) is important for neurotransmission (2). The third NOS (inducible NOS; iNOS) is induced in macrophages and other cells by soluble stimuli such as bacterial lipopolysaccharide (LPS) and by particulate stimuli such as zymosan (3), both in the presence of cytokines such as interferon gamma (IFN- $\gamma$ ).

Nitric oxide produced by the inducible NOS has an important role in the cytotoxicity of activated macrophages against several parasites and tumor cells $(2,4,5)$. Indeed, $N O$ 
has been shown to kill several pathogenic agents such as fungi (Cryptococcus neoformans (4); Candida albicans (6)), mycobacteria (7) and some intracellular protozoan parasites, such as Toxoplasma gondii (8), Leishmania major (9) and Trypanosoma cruzi (10). Cytotoxic effects of NO on an extracellular Platyhelminth parasite, Schistosoma mansoni, have also been reported (11). More recently, some reports indicated that peroxynitrite, the product of the reaction between NO and superoxide anion (12), may be the cytotoxic effector responsible for the killing of several targets $(13,14)$.

The most prevalent cause of diarrhea in the world is the infection of the upper gastrointestinal tract by the protozoan Giardia lamblia (15). The infection can be life-threatening in undernourished children of Third World countries and in immunocompromised patients (15).

In the present report, we show that NO produced by either activated macrophages or NO donors is cytotoxic to Giardia lamblia trophozoites. This effect can be attributable to NO alone and does not require the participation of either reactive oxygen intermediates or peroxynitrite.

\section{Material and Methods}

\section{Material}

Murine recombinant interferon- $\gamma$ was from Genzyme, (Cambridge, MA). LPS from Salmonella typhosa (0901) was obtained from Difco (Detroit, MI). S-Nitroso-acetyl-penicillamine (SNAP) and N-iminoethyl-L-ornithine (L-NIO) were kindly provided by Dr. S. Moncada, Wellcome Research Laboratories (Beckenham, UK). Zymosan, superoxide dismutase (SOD; from bovine erythrocytes), catalase and sodium nitroprusside (SNP) were from Sigma (St. Louis, MO). 3-Morpholinosydnonimine hydrochloride (SIN-1) waskindly provided by Dr. R. Henning, Cassela AG, Frankfurt, Germany. Fetal calf serum (FCS) was from Fazenda Pigue (Rio de Janeiro, RJ,
Brazil). All other tissue culture reagents were purchased from Sigma.

\section{Macrophages}

Murine peritoneal macrophages were harvested from C57/Black6 mice which had been injected ip 4 days before with $2 \mathrm{ml}$ of sterile thioglycollate solution $(3 \% \mathrm{w} / \mathrm{v}$ in water). Cells were plated onto 48 -well plates at $10^{6}$ cells/well, incubated for $2 \mathrm{~h}$ in $5 \% \mathrm{CO}_{2}$ at $37^{\circ} \mathrm{C}$ and washed with fresh medium to remove non-adherent cells. Assays were carried out in RPMI-1640 supplemented with $10 \%$ FCS, $20 \mathrm{mM}$ HEPES, $2 \mathrm{mM} \mathrm{L-}$ glutamine, $100 \mathrm{U} / \mathrm{ml}$ penicillin and $100 \mu \mathrm{g} /$ $\mathrm{ml}$ streptomycin.

\section{Giardia lamblia cultures}

Trophozoites of the Portland I strain (P1, ATCC-30889) were kept in 15-ml plastic tubes with $14 \mathrm{ml}$ TYI-S-33 medium supplemented with $0.1 \%$ bovine bile and $10 \%$ FCS (16). The medium was removed twice a week by aspiration and fresh medium was added to the tubes. For experiments, tubes containing the organisms were kept in ice-cold water for $20 \mathrm{~min}$ and centrifuged $(250 \mathrm{~g}, 10$ $\min$ ) in the cold. With this procedure more than $95 \%$ of the parasites detached from the tube and could be recovered in the pellet.

\section{Macrophage activation}

Macrophage activation was induced by adding IFN- $\gamma(20 \mathrm{U} / \mathrm{ml})$ plus LPS (100 ng/ $\mathrm{ml}$; referred to as LI) or the same concentration of IFN- $\gamma$ plus zymosan $(80 \mu \mathrm{g} / \mathrm{ml}$; referred to as ZI) to the macrophages. NO production was assessed by nitrite concentrations in the medium using the Griess reaction (17). Briefly, $100 \mu \mathrm{l} \mathrm{Griess} \mathrm{reagent} \mathrm{(equal}$ parts of $1 \% \mathrm{w} / \mathrm{v}$ sulfanilamide in $5 \% \mathrm{H}_{3} \mathrm{PO}_{4}$ and $0.1 \% \mathrm{w} / \mathrm{v}$ naphthylethylene diamine dihydrochloride in water) was added to 100 $\mu l$ of each supernatant in a 96-well plate. The 
plates were read using a Tytertek MKI340P plate reader at $540 \mathrm{~nm}$ against a standard curve of sodium nitrite in culture medium.

\section{Giardicidal assay}

Peritoneal macrophages were activated as described above and $12 \mathrm{~h}$ later, at the peak of NO synthase activity (18), Giardia trophozoites were added at a ratio of $4: 1$ (effector:target). The plate was then further incubated for $2 \mathrm{~h}$ at $37^{\circ} \mathrm{C}$ in $5 \% \mathrm{CO}_{2}$ and transferred to a refrigerator $\left(4^{\circ} \mathrm{C}\right)$ for $30 \mathrm{~min}$ to detach trophozoites. The suspension was centrifuged, supernatants were saved for nitrite assay and the pellet was resuspended in cold Trypan blue $(0.1 \%)$ to count viable parasites. As there is a good correlation between dye uptake and parasite motility, we evaluated parasite viability by these two criteria, ranking as dead parasites only those that took up the dye and were not moving. When NO donors were used they were incubated at different concentrations with $1 \times 10^{6}$ Giardia trophozoites/ml in RPMI medium for $2 \mathrm{~h}$ at $37^{\circ} \mathrm{C}$. The tubes were chilled and centrifuged and the pellet was resuspended in cold Trypan blue as described above. The results are reported as percent viable Giardia trophozoites in a total count of 200 parasites. Addition of NO donors did not change the $\mathrm{pH}$ of the incubation medium.

\section{Peroxynitrite synthesis}

Peroxynitrite was synthesized according to Beckman et al. (19). The concentrations of peroxynitrite used in the killing assays did not modify the $\mathrm{pH}$ of the medium. When tested as a bactericidal agent against $E$. coli, peroxynitrite prepared in our laboratory had an $\mathrm{LD}_{50}$ of about $250 \mu \mathrm{M}$, identical to the value obtained by Zhu et al. (14).

\section{Statistical analysis}

Results are reported as mean \pm SD. Dif-
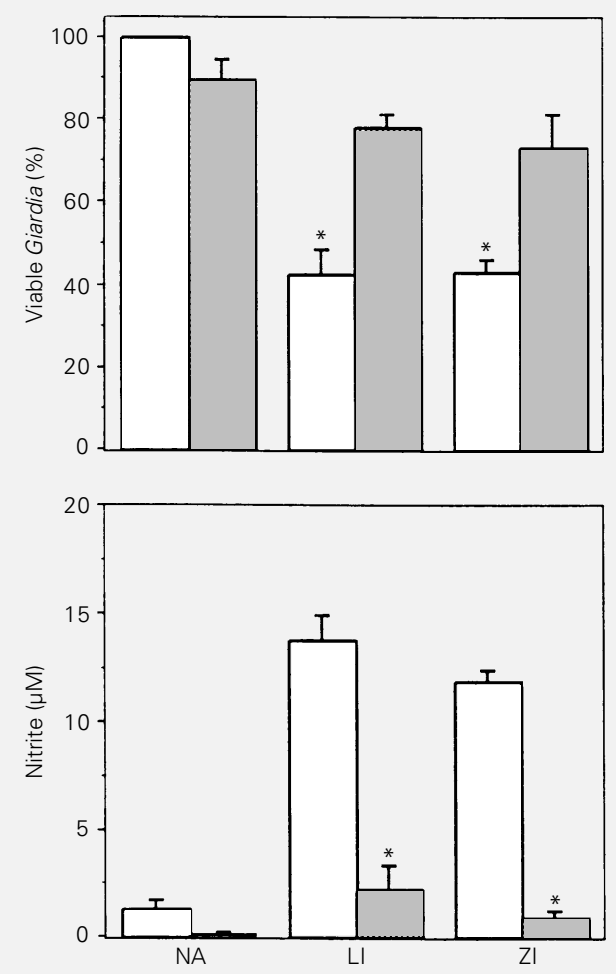

ferences were considered to be significant when $\mathrm{P}<0.05$, using analysis of variance followed by the Bonferroni $t$-test.

\section{Results}

Activation of mouse peritoneal macrophages with ZI or LI induced NO synthase activity with production of $\mathrm{NO}$ as assessed by increased nitrite levels in the supernatants (Figure 1, open bars). It is important to point out that the nitrite concentrations shown in Figure 1 represent the accumulation over a period of $2 \mathrm{~h}$ of exposure to Giardia trophozoites. When measured $48 \mathrm{~h}$ after activation, nitrite concentrations were $3 \pm 1$ and $80 \pm 5$ $\mu \mathrm{M}(\mathrm{N}=3)$ in non-activated and activated macrophages, respectively. That NO was responsible for the killing of Giardia trophozoites by activated macrophages was confirmed by showing that the NO synthase inhibitor L-NIO $(200 \mu \mathrm{M})$ inhibited both killing and nitrite accumulation (Figure 1, shaded bars). We also tested longer times of
Figure 1 - Effect of macrophage activation on Giardia trophozoite killing (upper panel) and nitrite production (lower panel). Murine peritoneal macrophages were activated with IFN- $\gamma(20 \mathrm{U} / \mathrm{ml})$ plus LPS (100 ng/ml; LI) or with IFN- $\gamma$ plus zymosan $(80 \mu \mathrm{g} / \mathrm{ml}$; ZI). Open and shaded bars are experiments performed in the absence or in the presence of $\mathrm{L}$ NIO $(200 \mu \mathrm{M})$, respectively. Twelve hours after macrophage activation, cells were washed with fresh medium, Giardia trophozoites were added at a ratio of 1:4 (target:effector; see Methods for details) and incubated for $2 \mathrm{~h}$. Supernatants were removed for nitrite determination and the pellet was used for the Giardiakilling assay. Results are reported as means \pm SD of triplicates. ${ }^{*} P<0.05$ when compared to non-activated cells (upper panel) or when activated cells in the absence of L-NIO were compared to cells in the presence of L-NIO (lower panel) (analysis of variance followed by the Bonferroni t-test). NA, Non-activated macrophages. The same results were obtained in two experiments. 
Figure 2 - Effects of increasing concentrations of $\mathrm{NO}$ donors on Giardia trophozoite killing. Giardiakilling was measured by incubating trophozoites with SNP (C), SNAP (-) or SIN-1 ( $\mathbf{\Delta})$ at the indicated concentrations for $2 \mathrm{~h}$ at $37^{\circ} \mathrm{C}$. Results are reported as means \pm SD of triplicates. The same results were obtained in three experiments.
Figure 3 - Enhancement of the Giardia trophozoite-killing effects of SIN-1 by superoxide dismutase. Trophozoites were incubated with increasing concentrations of SIN-1 in the absence (open bars) or in the presence (shaded bars) of SOD $(300 \mathrm{U} / \mathrm{ml}$ ) for $2 \mathrm{~h}$ at $37^{\circ} \mathrm{C}$. Results are reported as means \pm SD of triplicates. ${ }^{*} \mathrm{P}<0.05$ when compared to groups without SOD (analysis of variance followed by the Bonferroni $t$-test). The same results were obtained in two experiments. exposure of trophozoites to activated macrophages. Although it seems that increases in exposure times did not cause higher trophozoite killing (data not shown), these experiments were biased due to the great increase in trophozoite mortality even in the absence
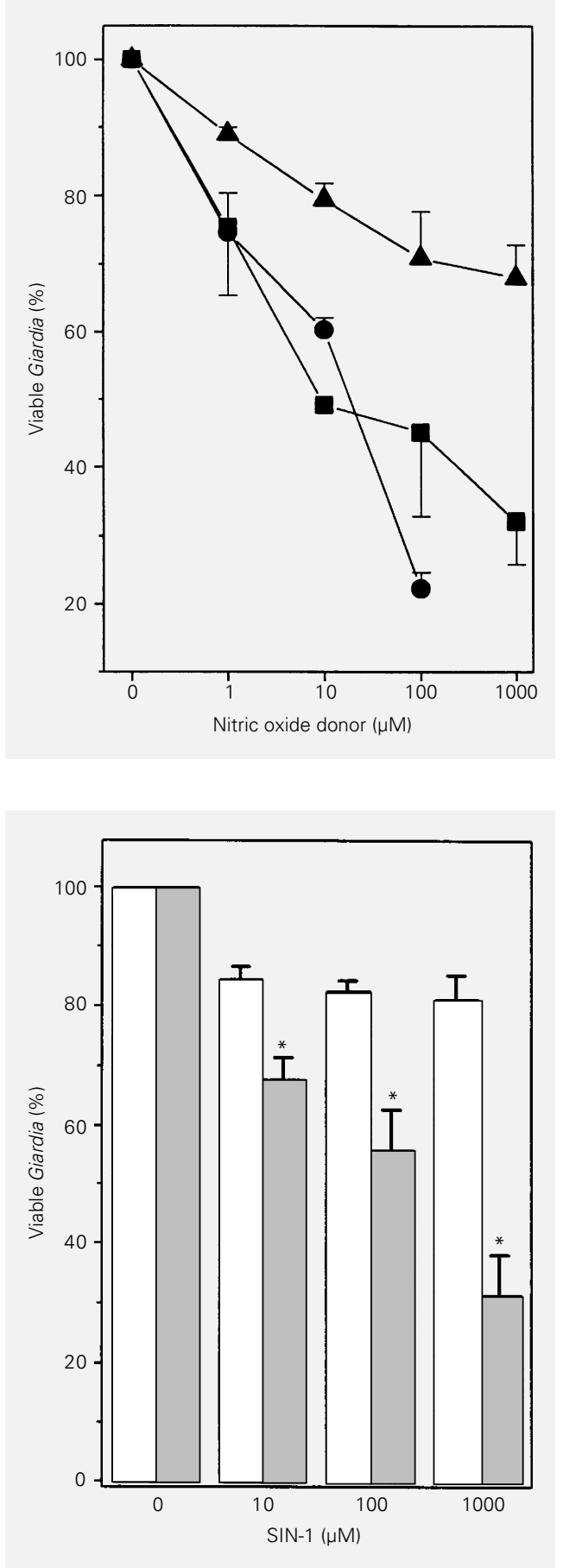

of macrophages when kept in a culture medium other than TYI-S-33 for more than 3-4 h. Presumably, this is attributable to the high cysteine concentration in Giardia medium which is critical to ensure high trophozoite viability (15).

To confirm that NO was responsible for the killing we tested for the effects of $\mathrm{NO}$ generated by NO donors on Giardia trophozoites in a macrophage-free system. When added directly to Giardia trophozoites, SNP was the most potent cytotoxic agent $\left(\mathrm{LD}_{50}\right.$ $\sim 50 \mu \mathrm{M})$, followed by SNAP $\left(\mathrm{LD}_{50} \sim 300\right.$ $\mu \mathrm{M})$ whereas SIN-1 was almost ineffective in this regard (Figure 2).

The presence of SOD did not change the killing effect of SNP or SNAP (data not shown). However, SOD greatly enhanced the effect of SIN-1. For instance, $100 \mu \mathrm{M}$ SIN-1 killed $17.5 \%$ and $57 \%$ of trophozoites in the absence and in the presence of SOD, respectively (Figure 3). We also performed a set of experiments in which trophozoites were incubated in medium containing SIN1 , SOD and catalase $(300 \mathrm{U} / \mathrm{ml})$. The presence of catalase abolished the potentiation of the cytotoxic effect of SIN-1 produced by SOD (SIN-1 alone: $47.3 \pm 0.7 \%$; SIN-1 + SOD: $24.5 \pm 3.3 \%$ and SIN-1 + SOD + catalase: $38.6 \pm 0.9 \%$ of viable trophozoites, $\mathrm{N}=3$ ).

In order to test the possibility that peroxynitrite is the cytotoxic effector against Giardia trophozoites, we examined the effects of the simultaneous incubation of the NO donor SNAP and the superoxide-forming system (xanthine/xanthine oxidase). This procedure is likely to yield peroxynitrite. The xanthine/xanthine oxidase system killed (although not significantly) a small number of Giardia trophozoites. In contrast, SNAP $(200 \mu \mathrm{M})$ killed $56.2 \%$ of the trophozoites (Figure 4, open bars). In the presence of superoxide, however, the cytotoxic effect of SNAP was completely abolished together with a decrease in nitrite levels (Figure 4, shaded bars). Similar results were obtained 
when the NO donor was SNP (data not shown). Authentic peroxynitrite was cytotoxic to Giardia trophozoites but only at very high concentrations (Figure 5) when compared to SNP, SNAP or SIN-1 (the latter in the presence of SOD).

\section{Discussion}

The induction of NO production in peritoneal macrophages by either soluble (LI) or particulate (ZI) stimuli turned these cells into cytotoxic effectors against Giardia trophozoites. This effect was highly dependent on NO production as L-NIO, an NOS inhibitor, blocked both nitrite accumulation and trophozoite killing. Similar results have been reported for Leishmania killing by activated macrophages (20).

NO has been shown to be cytotoxic for a number of parasites (5), but in view of the demonstration that $\mathrm{NO}$ can react with superoxide anion to yield peroxynitrite (12), which is also cytotoxic to some organisms, the identity of the effector species remains controversial. As shown by the present results, NO alone accounts for cytotoxicity against Giardia trophozoites. First, the addition of an NO synthase inhibitor to activated macrophages completely inhibited trophozoite killing. Second, the NO donors SNAP and SNP reproduced the killing effect observed with activated macrophages, and this effect was not changed in the presence of SOD. Third, incubation of SNAP or SNP together with a superoxide anion producer (xanthine/xanthine oxidase), a procedure likely to yield peroxynitrite, dramatically reduced the cytotoxic effect of NO donors. Grisham and Miles (21) have shown that a maximal yield of peroxynitrite is obtained only when NO and superoxide anion react at equimolar concentrations. If one admits that all $\mathrm{NO}$ released by $200 \mu \mathrm{M}$ of an NO donor reacts with superoxide, this would release $200 \mu \mathrm{M}$ peroxynitrite. Consistent with the conclusion that peroxynitrite is less cytotoxic for

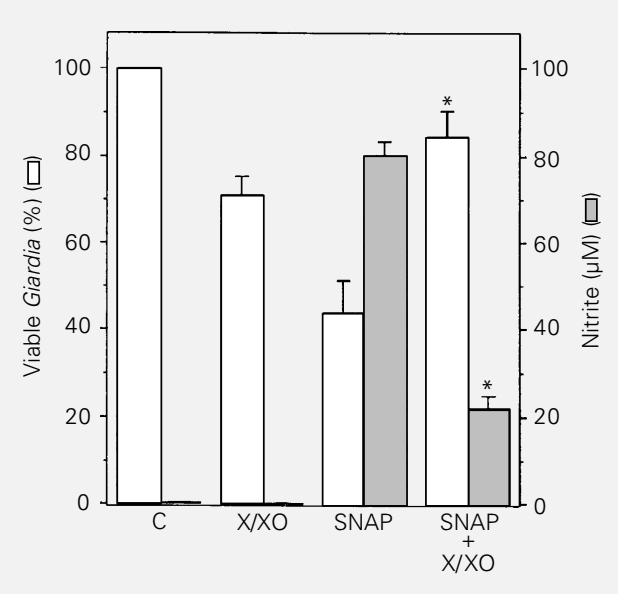

Figure 4 - Effects of SNAP, xanthine/xanthine oxidase (X/XO) system or both on Giardia trophozoite killing (open bars) and nitrite accumulation (shaded bars). Trophozoites were incubated either with SNAP (200 $\mu \mathrm{M})$, xanthine $(1 \mathrm{mM}) /$ xanthine oxidase $(20 \mathrm{mU} / \mathrm{ml})$ or both for 2 $\mathrm{h}$ at $37^{\circ} \mathrm{C}$. Results are reported as means \pm SD of triplicates. ${ }^{*} P<0.05$ when compared to the effects of SNAP alone (analysis of variance followed by the Bonferroni $t$-test). The same results were obtained in two experiments. C, Control.

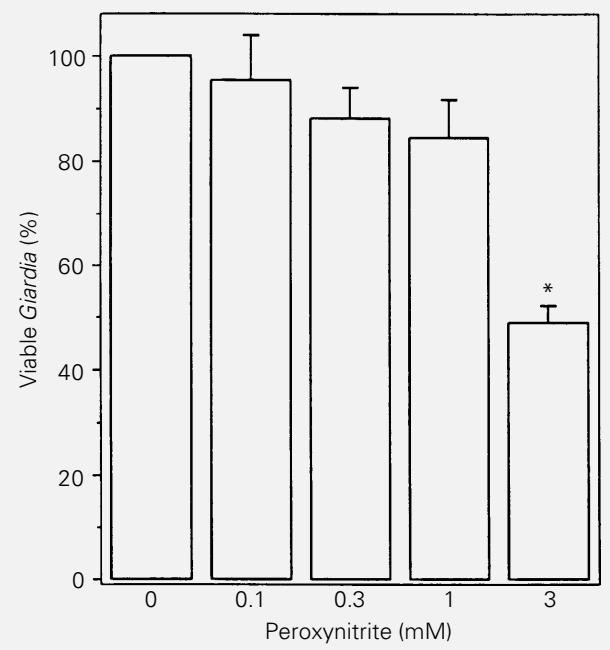

Figure 5 - Effect of increasing concentrations of authentic peroxynitrite on Giardia trophozoite killing. Peroxynitrite anion was obtained as described in the Methods section and incubated with trophozoites for $2 \mathrm{~h}$ at $37^{\circ} \mathrm{C}$. Addition of peroxynitrite did not change medium $\mathrm{pH}$. Results are reported as means \pm SD of triplicates. ${ }^{*} P<0.05$ when compared to the group without peroxynitrite (analysis of variance followed by the Bonferroni $t$-test). The same results were obtained in two experiments.
Giardia than NO, we have found that authentic peroxynitrite exhibited some cytotoxic effects only at high concentrations (3 $\mathrm{mM})$. Sensitivity to peroxynitrite seems to vary among microorganisms since peroxynitrite is an effective cytotoxic agent for Escherichia coli (14) and Trypanosoma cruzi (13), but not for Leishmania (20) or for Giardia (present study). It may be that in physiological situations, defense cells could use either NO or peroxynitrite as cytotoxic molecules, depending on the kinetics of production of superoxide anion and NO. For instance, human neutrophils stimulated with 
phorbol esters produce NO and superoxide with very similar kinetics, thus favoring peroxynitrite formation (22). On the other hand, in mouse macrophages activated by zymosan, superoxide is produced several hours earlier than NO, yet these cells potently killed Leishmania. In this system, peroxynitrite formation is unlikely to occur (20).

SIN-1, which is reported to release both NO and superoxide anion simultaneously (23) and therefore to yield peroxynitrite, exhibited a modest trophozoite-killing effect. However, superoxide removal (achieved by using SOD) greatly enhanced the cytotoxic effect of SIN-1. This enhancement, however, was blunted by catalase. It was reported that SOD enhancement of the SIN1 cytotoxic effect on a human liver cell line was dependent on hydrogen peroxide resulting from dismutation of superoxide and not on increased NO release (24). These investigators showed that the cytotoxic species are not NO, peroxynitrite or hydroxyl radical $(\cdot \mathrm{OH})$. Indeed, this seems to be the case in our experiments with SIN-1, since catalase inhibited the SOD-enhancing trophozoitekilling effect of SIN-1. Until this matter is addressed in more detail, one should be careful in interpreting the effect of SIN-1 in the presence of SOD. This point, however, seems to be relevant only for SIN-1, as the addition of SOD and/or catalase did not have any effect on the ability of activated macrophages or SNAP to kill Giardia trophozoites (data not shown).

Reactive oxygen species seem to be important for the killing of some intracellular parasites. Although activated macrophages are able to produce toxic reactive oxygen species (during phagocytosis, for example), this seems not to be the case for Giardia killing, as the parasite is too big (15) to be ingested by the macrophage. Therefore, in an in vivo infection, activated macrophages should use an easily diffusible chemical species to exert their killing action, as the majority of the target will be extracellular. The following lines of evidence point to $\mathrm{NO}$ as this effector: i) the almost complete inhibition of trophozoite killing by activated macrophages when an NO synthase inhibitor was present in the medium (Figure 1); ii) superoxide anion does not easily cross membranes (25); iii) hydrogen peroxide does (25), and catalase does not interfere with the killing caused by activated macrophages or SNAP (data not shown); iv) peroxynitrite has a very short half-life and is inactivated by physiological concentrations of bicarbonate (>25 mM; Ref. 14). In summary, from the vast array of cytotoxic molecules potentially produced by defense cells, NO seems to account for the majority, if not all, of macrophage giardicidal effects.

\section{Acknowledgments}

We appreciate the technical assistance of Vicente Conrado Leite. We thank Dr. S. Moncada (Wellcome, UK) for the gift of SNAP and L-NIO, Dr. R. Henning (Cassela AG, Germany) for the gift of SIN-1, Dr. W.M. Kattenbach (UERJ, Brazil) for the gift of and helpful assistance in growing and maintaining Giardia lamblia parasites, and Dr. V. Koatz (UFRJ, Brazil) for allowing us to use her tissue culture facilities. We also thank Dr. M.M. Sorenson for her critical review of the manuscript. 


\section{References}

1. Nathan CF (1980). Secretory products of macrophages. Journal of Clinical Investigation, 79: 319-326.

2. Moncada S, Palmer RMJ \& Higgs EA (1991). Nitric oxide: physiology, pathophysiology, and pharmacology. Pharmacological Reviews, 43: 109-142.

3. Cunha FQ, Assreuy J, Liew FY \& Moncada $S$ (1993). Phagocytosis and induction of nitric oxide synthase in murine macrophages. Immunology, 79: 408-411.

4. Granger DL, Hibbs JB, Perfect JR \& Durack DT (1990). Metabolic fate of Larginine in relation to microbiostatic capability of macrophages. Journal of Clinical Investigation, 85: 264-273.

5. Liew FY (1993). The role of nitric oxide in parasitic diseases. Annals of Tropical Medicine and Parasitology, 87: 637-642.

6. Cenci E, Romani L, Mencacci A, Spaccapelo R, Chiraffella E, Puccetti P \& Bistoni $F$ (1993). Interleukin-4 and interleukin-10 inhibit nitric oxide-dependent macrophage killing of Candida albicans. European Journal of Immunology, 23: 1034-1038.

7. Chan J, Xing Y, Magliozzo RS \& Bloom BR (1992). Killing of virulent Mycobacterium tuberculosis by reactive nitrogen intermediates produced by activated murine macrophages. Journal of Experimental Medicine, 175: 1111-1122.

8. Adams L, Hibbs JB, Taintor RR \& Krahenbuhl JL (1990). Microbiostatic effect of murine-activated macrophages for Toxoplasma gondii. Role for synthesis of inorganic nitrogen oxides from L-arginine. Journal of Immunology, 144: 2725-2729.

9. Liew FY, Millot S, Parkinson C, Palmer RMJ \& Moncada S (1990). Macrophage killing of Leishmania parasite in vivo is mediated by nitric oxide from L-arginine. Journal of Immunology, 144: 4794-4797.
10. Munoz-Fernandez MA, Fernandez M \& Fresno M (1992). Synergism between tumor necrosis factor- $\alpha$ and interferon- $\gamma$ on macrophage activation for the killing of intracellular Trypanosoma cruzi through a nitric oxide-dependent mechanism. European Journal of Immunology, 22: 301-307.

11. James SL \& Glaven J (1989). Macrophage cytotoxicity against schistosomula of Schistosoma mansoni involves argininedependent production of reactive nitrogen intermediates. Journal of Immunology, 143: 4208-4212.

12. Beckman JS, Beckman TW, Chen J, Marshall PA \& Freeman BA (1990). Apparent hydroxyl radical production by peroxynitrite: implications for endothelial injury from nitric oxide and superoxide. Proceedings of the National Academy of Sciences, USA, 87: 1620-1624.

13. Denicola A, Rubbo H, Rodriguez D \& Radi R (1993). Peroxynitrite-mediated cytotoxicity to Trypanosoma cruzi. Archives of Biochemistry and Biophysics, 304: 279286.

14. Zhu L, Gunn C \& Beckman JS (1992). Bactericidal activity of peroxynitrite. Archives of Biochemistry and Biophysics, 298: 452-457.

15. Adam RD (1991). The biology of Giardia spp. Microbiological Reviews, 55: 706732.

16. Keister DB (1983). Axenic culture of Giardia lamblia in TYI-S-33 medium supplemented with bile. Transactions of the Royal Society of Tropical Medicine and Hygiene, 77: 487-488.

17. Schmidt HHHW, Wilke $P$, Evers $P$ \& Bohme E (1989). Enzymatic formation of nitrogen oxides from L-arginine in bovine brain cytosol. Biochemical and Biophysical Research Communications, 165: 284291.
18. Assreuy J, Cunha FQ, Liew FY \& Moncada $S$ (1992). Feedback inhibition of nitric oxide synthase by nitric oxide. British Journal of Pharmacology, 108: 833-837.

19. Beckman JS, Chen J, Ischiropoulos H \& Crow JP (1994). Oxidative chemistry of peroxynitrite. Methods in Enzymology, 233: 229-240

20. Assreuy J, Cunha FQ, Epperlein M Noronha-Dutra A, O'Donnell CA, Liew FY \& Moncada S (1994). Production of nitric oxide and superoxide by activated macrophages and killing of Leishmania major. European Journal of Immunology, 24: 672-676.

21. Grisham MB \& Miles AM (1995). Interaction between superoxide and nitric oxide under physiological conditions. Endothelium, 3 (Suppl): S6 (Abstract).

22. Carreras MC, Pargament GA, Catz SD, Poderoso JJ \& Boveris A (1994). Kinetics of nitric oxide and hydrogen peroxide production and formation of peroxynitrite during the respiratory burst of human neutrophils. FEBS Letters, 341: 65-68.

23. Hogg N, Darley-Usmar VM, Wilson MT \& Moncada S (1992). Production of hydroxyl radicals from the simultaneous generation of superoxide and nitric oxide. Biochemical Journal, 281: 419-424.

24. Gergel D, Misik V, Ondrias K \& Cederbaum Al (1995). Increased cytotoxicity of 3-morpholinosydnonimine to HepG2 cells in the presence of superoxide dismutase. Role of hydrogen peroxide and iron. Journal of Biological Chemistry, 270: 20922-20929.

25. Halliwell B (1992). Reactive oxygen species and the central nervous system. Journal of Neurochemistry, 59: 1609-1623. 